\title{
Instanton contribution to the quark form factor
}

\author{
Alexander E. Dorokhov* \\ Bogolyubov Laboratory of Theoretical Physics, Joint Institute for Nuclear Research, 141980 Dubna, Russia \\ Igor O. Cherednikov ${ }^{\dagger}$ \\ Bogolyubov Laboratory of Theoretical Physics, Joint Institute for Nuclear Research, 141980 Dubna, Russia \\ and Institute for Theoretical Problems of Microphysics, Moscow State University, 119899 Moscow, Russia
}

(Received 16 April 2002; published 16 October 2002)

\begin{abstract}
The nonperturbative effects in the quark form factor are considered in the Wilson loop formalism. The properties of the Wilson loops with cusp singularities are studied taking into account the perturbative and nonperturbative contributions, where the latter are considered within the framework of the instanton liquid model. For the integration path corresponding to this form factor-the angle with infinite sides - the explicit expression for the vacuum expectation value of the Wilson operator is found to leading order. The calculations are performed in the weak-field limit for the instanton vacuum contribution and compared with the one- and two-loop order results for the perturbative part. It is shown that the instantons produce the powerlike corrections to the perturbative result, which are comparable in magnitude with the perturbative part at the scale of order of the inverse average instanton size. It is demonstrated that the instanton contributions to the quark form factor are exponentiated to high orders in the small instanton density parameter.
\end{abstract}

DOI: 10.1103/PhysRevD.66.074009

PACS number(s): 12.38.Lg, 11.10.Gh

\section{INTRODUCTION}

A convenient approach which may be applied to the investigation of the infrared behavior of QCD is the formulation of the non-Abelian gauge theory on loop space that becomes possible by virtue of the close correspondence between gauge and chiral fields [1]. One of the advantages of this method is that it allows one to consider both the perturbative and nonperturbative contributions. The basic object of study in such an approach is the gauge invariant vacuum average of the Wilson loop operator.

$$
W(C)=\frac{1}{N_{c}} \operatorname{Tr}\left\langle 0\left|\mathcal{P} \exp \left(i g \int_{C} d x_{\mu} \hat{A}_{\mu}(x)\right)\right| 0\right\rangle,
$$

where the integration goes along the closed contour $C$ and the gauge field

$$
\hat{A}_{\mu}(x)=T^{a} A_{\mu}^{a}(x), \quad T^{a}=\frac{\lambda^{a}}{2},
$$

belongs to the Lie algebra of the gauge group $S U\left(N_{c}\right)$, while the Wilson loop operator $\mathcal{P} e^{i g \int d x A(x)}$ lies (for quark lines) in its fundamental representation.

In particular, the objects such as path-ordered contour integrals of the gauge field emerge naturally in the study of hard collisions of hadrons (Drell-Yan process, deep inelastic scattering, etc.) and the hadron form factors in the eikonal approximation $[2,3]$. The use of the Wilson integral formalism in the heavy quark effective theory has also been discussed (see, e.g., Ref. [4]). In the case of hard processes, the integration contours become infinite, since they present the

\footnotetext{
*Email address: dorokhov@thsun1.jinr.ru

†Email address: Igor.Cherednikov@jinr.ru
}

classical trajectories of the partons participating in the collision. Considering the parton scattering processes at large total energies and small momentum transfers it is revealed that the role of the resumed soft gluons exchanges becomes significant. While in QED this problem is solved in closed form yielding the well-known Sudakov form factor (by virtue of exponentiation of the leading one loop perturbative term), in QCD the situation appears to be more complicated due to its non-Abelian nature and the influence of original nonperturbative effects. Nevertheless, it has been shown in perturbation theory that in QCD processes the exponentiation takes place with the exponent given by a series in the strong coupling constant. Additional soft contributions may appear due to various nonperturbative effects, a number of which are closely related to the nontrivial structure of the QCD vacuum. In the case of the instanton ensemble modeling the QCD vacuum, the exponentiation of the single instanton contribution can also be proven (see Sec. IV). Indeed, although the QCD vacuum plays an important role in the high-energy collisions, the direct investigation of these effects remains a difficult task. The aim of the present work is to evaluate them within the framework of the instanton liquid model of QCD vacuum for the simplest possible quantity - the quark form factor.

It is generally believed that the nonperturbative structure of QCD can be well understood within the instanton liquid model [5]. Considering the QCD vacuum as an ensemble of instantons, one can describe a lot of the low-energy phenomena in strong interactions on the qualitative, as well as the quantitative level. The importance of the instanton induced effects of strong interactions is also supported by lattice simulations $[5,6]$. The instanton picture is generally considered as a fruitful and perspective framework for hadronic physics. The role of the instantons in hard hadronic processes has been studied intensively, both theoretically and experimentally. The perspectives for an unambiguous experimental 
detection of instanton contributions are believed to be optimistic and very promising. The main problem arising in this context is the evaluation of instanton induced effects at a hard scale of a given process [7]. Hence, we are going to study the instanton induced contributions to the Wilson integral over contours of a various geometry and apply the obtained results to the analysis of nonperturbative effects in the high-energy hadron collisions. Recently a similar approach has been developed in the study of high energy elastic and quasielastic parton-parton scattering [8]. The vacuum averages of Wilson operators with finite-length contours were considered in the instanton model in Ref. [9], where the properties of the nonlocal quark and gluon condensates were discussed.

In the present paper, we start the investigation of the instanton induced effects in the high-energy QCD processes. We propose an approach which allows one to evaluate the instanton contributions to the Wilson integrals made of several (in)finite lines containing specific cusp and/or cross singularities. For this purpose, we start with one of the simplest configurations, i.e., the angle with infinite sides that corresponds to the integration path for the Wilson operator describing the soft part of the quark Sudakov form factor [10]. Such configurations enter as constructive units in the Wilson contours for many high energy processes.

This paper is organized as follows. In Sec. II, we briefly describe the general renormalization properties of the Wilson loops with cusp singularities and find the explicit formula in leading order, applicable both to perturbative and nonperturbative fields. Then we analyze the UV and IR divergencies and calculate the perturbative part in an arbitrary covariant gauge demonstrating explicitly the gauge invariance of the obtained (renormalized) result. Section III is devoted to the study of the instanton induced nonperturbative contributions. The expression for them is found in the closed form in the weak-field limit in terms of the instanton profile function. It is shown that the instantons actually give powerlike corrections to the perturbative result. The magnitude of the instanton induced effects is found to be comparable with the perturbative part at a certain low momentum scale. In Sec. IV, we prove the exponentiation in the small instanton density parameter of the all-order single instanton contribution to the Sudakov form factor. Afterwards, we discuss the possible applications of the results obtained above to investigations of the physically interesting processes and make some conclusive remarks.

\section{RENORMALIZATION AND PERTURBATIVE CONTRIBUTION}

Let us consider in short the renormalization properties of the Wilson loop. It is well known, that evaluation of $W(C)$ (1) encounters divergencies. The divergencies appearing for smooth contours without self-intersections can be removed by means of the convenient $R$ operation [11,12]

$$
W_{R}\left(C, g_{R}, \mu\right)=\lim _{\varepsilon \rightarrow 0} \widetilde{W}\left(C, g_{R}, \mu, \varepsilon\right) \equiv \lim _{\varepsilon \rightarrow 0} R W(C, g, \varepsilon),
$$

where $\varepsilon$ is the dimensional regularization parameter and $\mu$ is the UV normalization scale. In this case, the $R$ operation in the multiplicative renormalization consists of the coupling constant $g \rightarrow g_{R}$ and the field $\hat{A}_{\mu} \rightarrow \hat{A}_{\mu}^{R}$.

In the more general case, the contour may contain a number of cusps, i.e., the points where the derivative is not smooth. In this situation, divergencies of another type arise which depend on the corresponding cusp angles $\gamma_{i}$. Although they were considered as a shortcoming of the loop formulation of a gauge theory about two decades ago, these singularities (and the anomalous dimensions related to them) are shown to play an important role in the partonic hard processes controlling the asymptotics of the scattering amplitudes [13]. In order to remove these divergencies, the generalized $K_{\gamma}$ operation has been proposed [12]. For a contour with one cusp $C_{\gamma}$ this operation is reduced to

$$
W_{R}\left(C_{\gamma}, g_{R}, \mu, \bar{C}_{\gamma}\right)=\lim _{\varepsilon \rightarrow 0} K_{\gamma} \widetilde{W}\left(C_{\gamma}, g_{R}, \mu, \varepsilon\right),
$$

where the contour $\bar{C}_{\gamma}$ is treated as a subtraction point. It has been proved that the $K_{\gamma} R$ operation renormalizes multiplicatively any loop integral with finite number of cusps $[12,14]$. The multiplicative renormalizability means that the $K_{\gamma}$ operation acts as

$$
K_{\gamma} \widetilde{W}\left(C_{\gamma}, g_{R}, \mu, \varepsilon\right)=Z_{\text {cusp }}\left(g_{R}, \gamma, \mu, \varepsilon\right) \widetilde{W}\left(C_{\gamma}, g_{R}, \mu, \varepsilon\right) \text {, }
$$

where

$$
Z_{\text {cusp }}=\widetilde{W}^{-1}\left(\bar{C}_{\gamma}, g_{R}, \mu, \varepsilon\right)=1+\sum_{n=1}^{\infty}\left(\frac{\alpha_{S}}{\pi}\right)^{n} Z_{n}(\gamma, \mu, \varepsilon)
$$

is an additional renormalization constant. The coefficients $Z_{n}$ are the sum of simple poles plus some finite terms

$$
Z_{n}(\gamma, \varepsilon)=\sum_{k=1}^{n}\left(\frac{1}{\varepsilon^{k}} A_{k n}(\gamma)\right)+(\text { finite terms })_{n} .
$$

A choice of the finite part of $Z_{n}$ to each order actually determines the $K_{\gamma}$ subtraction scheme. In order to remove pole terms we shall use the modified minimum subtraction $\overline{\mathrm{MS}}$-scheme $\left(K_{\gamma}^{\overline{\mathrm{MS}}}\right)$.

The first nontrivial cusp dependent term in the expansion of $W(C)$ (1) in powers of $g^{2}$ for the angle with two infinite straight line rays [Fig. 1(a)] contains the contributions from both perturbative [Fig. 1(b)] and nonperturbative [Fig. 1(c)] fields

$$
W^{(1)}(\gamma)=W_{P}^{(1)}(\gamma)+W_{\mathrm{NP}}^{(1)}(\gamma) .
$$

Regardless of which part is considered-either perturbative, or nonperturbative-we will write the one-loop contribution in the following form:

$$
W^{(1)}(\gamma)=-\frac{g^{2} C_{F}}{2} \int_{C_{\gamma}} d x_{\mu} \int_{C_{\gamma}} d y_{\nu} D_{\mu \nu}(x-y),
$$






(a)

(b)

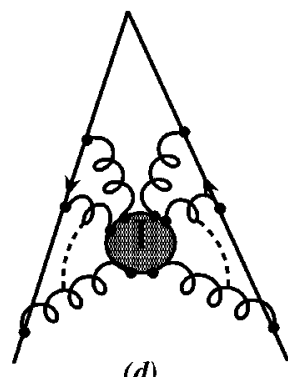

(d)

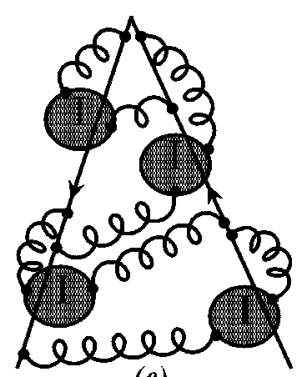

(e)

FIG. 1. The notations for the quark momenta and the total cuspdependent part of the Wilson loop integral for the quark form factor (a); the leading order contributions of the perturbative (b) and nonperturbative (single-instanton) (c) fields; (d) the all-order single instanton result; (e) the exponentiation of the single instanton result.

where $C_{F}=\left(N_{c}^{2}-1\right) / 2 N_{c}$. It is convenient to present the gluon propagator $D_{\mu \nu}(z)$ in the form

$$
D_{\mu \nu}(z)=\delta_{\mu \nu} \partial_{z}^{2} d_{1}\left(z^{2}\right)-\partial_{\mu} \partial_{\nu} d_{2}\left(z^{2}\right)
$$

Here and in what follows, we use the dimensional regularization with $n=4-2 \varepsilon, \varepsilon<0$ in order to control the IRdivergent terms in the integrals. The remaining UV singularity (due to the infinitely small $z^{2}$ in the vicinity of the cusp) will be regularized by the corresponding UV cutoff.

The trajectories of the incoming and outgoing quarks [Fig. 1(a)] may be parametrized as

$$
x=v_{1} s(0<s<\infty), \quad y=v_{2} \tau(-\infty<\tau<0) .
$$

The angle between the vectors $v_{1}$ and $v_{2}$ is given in the Minkowski space by

$$
\begin{gathered}
\cosh \chi=\left(v_{1} v_{2}\right)=\frac{\left(p_{1} p_{2}\right)}{m^{2}}=1+\frac{Q^{2}}{2 m^{2}}, \\
-Q^{2}=\left(p_{2}-p_{1}\right)^{2}, \quad v_{1,2}^{2}=1,
\end{gathered}
$$

where the quark momenta are supposed to be on shell: $p_{1}^{2}$ $=p_{2}^{2}=m^{2}$. The continuation to the Euclidean space is defined as $[14,15]$

$$
\chi \rightarrow i \gamma
$$

By virtue of the interpretation of the graph Fig. 1 as an amplitude of the elastic scattering of an on-mass-shell onedimensional fermion on a color singlet potential $[11,14]$, we have to consider the quantity

$$
W^{(1)}(\gamma)=\widetilde{W}^{1}(\gamma)-\widetilde{W}^{1}(0),
$$

where (for some technical details of the calculations, see the Appendix)

$$
\widetilde{W}^{1}(\gamma)=-g^{2} C_{F}\left[(n-2) d_{1}(0) \gamma \cot \gamma+d_{2}(0)\right] .
$$

It follows from Eq. (13) that the integrals (9) in which both points $x$ and $y$ belong to the same side of the angle do not contribute to the quantity $W^{(1)}(\gamma)$. Hence we have within the one loop accuracy

$$
W(\gamma)=1-4 \pi \alpha_{S} C_{F}(n-2) h(\gamma) d_{1}(0),
$$

where

$$
h(\gamma)=\gamma \cot \gamma-1
$$

is the universal cusp factor. We should emphasize here that the expression (15) holds for the perturbative part as well as for the nonperturbative part depending on the value $d_{1}(0)$. For the perturbative field, Eq. (15) reflects the explicit gauge invariance in the set of covariant gauges, since the gauge fixing parameter $\xi$ enters only in the function $d_{2}\left(z^{2}\right)$.

Let us consider first the perturbative part $W_{P}^{(1)}(\gamma)$. By using the free propagator in the Euclidean space (see the Appendix) the IR-regularized value of $d_{1}(0)$ can be written in the form

$$
d_{1}(0 ; \varepsilon, \lambda)=\frac{\left(\lambda^{2} \pi\right)^{\varepsilon}}{16 \pi^{2}} \int_{0}^{\infty} d \alpha \alpha^{-(1+\varepsilon)},
$$

where $\lambda^{2}$ is the IR regularization parameter. This integral diverges at the upper (UV) limit for $\varepsilon<0$, and hence we must regularize it. To this end, we may introduce the UV cutoff $\mu^{2}$, that corresponds to the following replacement in the denominator of the perturbative propagator $z^{2} \rightarrow z^{2}$ $+\mu^{-2}$, and finally we get

$$
d_{1}(0 ; \varepsilon, \mu / \lambda)=-\frac{1}{\varepsilon} \frac{1}{16 \pi^{2}}\left(\frac{\lambda^{2} \pi}{\mu^{2}}\right)^{\varepsilon}
$$

Thus one obtains, with one loop accuracy,

$$
W_{P}\left(\gamma, \alpha_{S}, \mu / \lambda, \varepsilon\right)=1+\frac{\alpha_{S}}{2 \pi} C_{F} h(\gamma) \frac{1}{\varepsilon}(1-\varepsilon)\left(\frac{\lambda^{2} \pi}{\mu^{2}}\right)^{\varepsilon},
$$

and the cusp renormalization constant $Z_{\text {cusp }}^{(1)}$ within the $\overline{\mathrm{MS}}$ scheme

$$
Z_{\text {cusp }}^{(1)}(\gamma, \varepsilon)=1-\frac{\alpha_{S}}{2 \pi} C_{F} h(\gamma)\left(\frac{1}{\varepsilon}+\ln \pi-1\right),
$$

which is in agreement with Refs. [16,12]. Therefore, the perturbative part of the finite renormalized function reads 


$$
\begin{aligned}
W_{P}(\gamma, \mu) & =\lim _{\varepsilon \rightarrow 0} Z_{\text {cusp }}^{(1)}(\gamma, \mu, \varepsilon) \widetilde{W}_{P}(\gamma, \mu, \varepsilon) \\
& =1-\frac{\alpha_{S}}{2 \pi} C_{F} h(\gamma) \ln \frac{\mu^{2}}{\lambda^{2}}
\end{aligned}
$$

The one-loop cusp anomalous dimension which satisfies the $\mathrm{RG}$ equation reads

$$
\begin{aligned}
& \left(\mu \frac{\partial}{\partial \mu}+\beta\left(g_{R}\right) \frac{\partial}{\partial g_{R}}\right) W_{P}\left[\gamma, \mu, \alpha_{S}(\mu)\right] \\
& \quad=-\Gamma_{P}^{\operatorname{cusp}_{P}^{(1)}}\left[\gamma, \alpha_{S}(\mu)\right] W_{P}\left[\gamma, \mu, \alpha_{S}(\mu)\right]
\end{aligned}
$$

where $\beta\left(g_{R}\right)$ is the usual QCD $\beta$ function $\beta\left(g_{R}\right)$ $=\mu(\partial / \partial \mu) g_{R}=$ const $g_{R}^{3}+O\left(g_{R}^{5}\right)$, which can be found in the one-loop order $[16,12]$

$$
\Gamma_{P}^{\mathrm{cusp}(1)}\left(\gamma, \alpha_{S}\right)=-\frac{d}{d \ln \mu} W_{P}^{(1)}(\gamma, \mu)=\frac{\alpha_{S}}{\pi} C_{F} h(\gamma) .
$$

Hence, we reproduce the Wilson operator value for the infinite contour with the Euclidean cusp parameter $\gamma$.

\section{INSTANTON CONTRIBUTION}

Let us estimate the nonperturbative contribution to $W(C)$ in the instanton model. The instanton field is given by

$$
\begin{aligned}
\hat{A}_{\mu}(x ; \rho) & =A_{\mu}^{a}(x ; \rho) \frac{\sigma^{a}}{2} \\
& =\frac{1}{g} \mathbf{R}^{a b} \sigma^{a} \eta_{\mu \nu}^{ \pm b}\left(x-z_{0}\right)_{\nu} \varphi\left(x-z_{0} ; \rho\right),
\end{aligned}
$$

where $\mathbf{R}^{a b}$ is the color orientation matrix $(a, b=1,2,3), \sigma^{a}$ 's are the Pauli matrices, and $( \pm)$ corresponds to the instanton or antiinstanton. The averaging of the Wilson operator over the nonperturbative vacuum is reduced to the integration over the coordinate of the instanton center $z_{0}$, the color orientation and the instanton size $\rho$. The measure for the averaging over the instanton ensemble reads $d I$ $=d \mathbf{R} d^{4} z_{0} d n(\rho)$, where $d \mathbf{R}$ refers to the averaging over color orientation, and $d n(\rho)$ depends on the choice of the instanton size distribution. Taking into account Eq. (24), we write the Wilson integral (1) in the single instanton approximation in the form

$$
w_{I}(C)=\frac{1}{N_{c}}\left\langle 0\left|\operatorname{Tr} \exp \left(i \sigma^{a} \phi^{a}\right)\right| 0\right\rangle,
$$

where the phase is

$$
\phi^{a}=\mathbf{R}^{a b} \eta^{ \pm b} \int_{C_{\gamma}} d x_{\mu}\left(x-z_{0}\right)_{\nu} \varphi\left(x-z_{0} ; \rho\right) .
$$

We omit the path ordering operator $\mathcal{P}$ in Eq. (25) because the instanton field (24) is a hedgehog in color space, and so it locks the color orientation by space coordinates. Performing the averaging over the color rotations, making use of the known property of 't Hooft symbols

$$
\eta_{\mu \nu}^{ \pm a} \eta_{\rho \sigma}^{ \pm a}=\delta_{\mu \rho} \delta_{\nu \sigma}-\delta_{\mu \sigma} \delta_{\nu \rho} \pm \varepsilon_{\mu \nu \rho \sigma},
$$

and performing the subtraction of self-energy part (13) we obtain the all-order single instanton contribution to the cuspdependent part of Wilson loop (1):

$$
w_{I}(\gamma)=\int d^{4} z_{0} \int d n(\rho)\left[\cos \phi\left(\gamma, z_{0}, \rho\right)-\cos \phi\left(0, z_{0}, \rho\right)\right],
$$

where the squared phase $\phi^{2}=\phi^{a} \phi^{a}$ may be written as

$$
\begin{aligned}
\phi^{2}\left(\gamma, z_{0}, \rho\right)= & \sum_{i, j=1,2}\left[\left(v_{i} v_{j}\right) z_{0}^{2}-\left(v_{i} z_{0}\right)\left(v_{j} z_{0}\right)\right] \\
& \times \int_{0}^{\infty} d \sigma \varphi\left\{\left[(-1)^{i+1} v_{i} \sigma-z_{0}\right]^{2} ; \rho\right\} \\
& \times \int_{0}^{\infty} d \sigma^{\prime} \varphi\left\{\left[(-1)^{j+1} v_{j} \sigma^{\prime}-z_{0}\right]^{2} ; \rho\right\} .
\end{aligned}
$$

Although the expressions (28), (29) give the complete formula for the all-order single instanton contribution, in what follows we restrict ourselves to the investigation of the weak field limit. Phenomenologically, it is assumed that the instanton size distribution is sharply peaked at a certain finite value. As we will see soon, this finite instanton size $\rho$ provides an UV cutoff for the nonperturbative gluon propagator. Therefore, for the instanton field we use the same dimensional IR regularization with $\varepsilon<0$ and the IR parameter $\lambda$ as in the perturbative case. ${ }^{1}$ So, in this limit the leading instanton induced term reads

$$
\begin{aligned}
w_{I}^{(1)}(\gamma)= & -\frac{g^{2} \lambda^{n-4}}{2} \int d n(\rho) \int_{C_{\gamma}} d x_{\mu} \int_{C_{\gamma}} d y_{\nu} \\
& \times \int \frac{d^{n} k}{(2 \pi)^{n}} \widetilde{A}_{\mu}^{a}(k ; \rho) \widetilde{A}_{\nu}^{a}(-k ; \rho) e^{-i k(x-y)}
\end{aligned}
$$

By using the Fourier transform of the instanton field

$$
\tilde{A}_{\mu}^{a}(k ; \rho)=-\frac{2 i}{g} \eta_{\mu \sigma}^{ \pm a} k_{\sigma} \tilde{\varphi}^{\prime}\left(k^{2} ; \rho\right),
$$

Eq. (30) can be written in the form of Eq. (10) with the instantonic analogue of the function $d_{1}\left(z^{2}\right)$ :

\footnotetext{
${ }^{1}$ Another possible approach to divergencies in the integrals over the instanton fields is to use a constrained instanton solution which is characterized by the profile function exponentially decreasing at large distances $[8,9]$.
} 


$$
d_{1}\left(z^{2}\right) \rightarrow d_{1}^{I}\left(z^{2}\right)=-\frac{1}{g^{2} C_{F}} \int d n(\rho) D_{I}\left(z^{2} ; \rho, \lambda\right)
$$

where

$$
D_{I}\left(z^{2} ; \rho, \lambda\right)=\lambda^{4-n} \int \frac{d^{n} k}{(2 \pi)^{n}} e^{-i k z}\left[2 \tilde{\varphi}^{\prime}\left(k^{2} ; \rho\right)\right]^{2} .
$$

Above, $\tilde{\varphi}\left(k^{2} ; \rho\right)$ is the Fourier transform of the instanton profile function $\varphi\left(z^{2} ; \rho\right)$ and $\tilde{\varphi}^{\prime}\left(k^{2} ; \rho\right)$ is its derivative with respect to $k^{2}$. Now using the result (15) of the previous section, we get the instanton contribution in the form

$$
w_{I}(\gamma ; \varepsilon, \lambda)=(n-2) h(\gamma) \int d n(\rho) D_{I}(0 ; \varepsilon, \lambda, \rho)
$$

Consider now the renormalization of the nonperturbative part for the instanton field in the singular gauge, ${ }^{2}$ where the profile function is

$$
\varphi(u ; \rho)=\frac{\rho^{2}}{z^{2}\left(z^{2}+\rho^{2}\right)} .
$$

By using the Fourier transform of this function one gets the IR dimensionally regularized $D_{I}(0 ; \varepsilon, \lambda, \rho)$ in the form

$$
D_{I}(0 ; \varepsilon, \lambda, \rho)=\frac{\rho^{4} \pi^{2+\varepsilon} \lambda^{2 \varepsilon}}{4} \int_{0}^{\infty} d \alpha_{1} d \alpha_{2} \int_{0}^{\infty} d \beta_{1} d \beta_{2} \frac{e^{-\rho^{2}\left(\alpha_{1}+\beta_{1}\right)}}{\left(\alpha_{1}+\alpha_{2}\right)^{1+\varepsilon}\left(\beta_{1}+\beta_{2}\right)^{1+\varepsilon}\left(\alpha_{1}+\alpha_{2}+\beta_{1}+\beta_{2}\right)^{2-\varepsilon}} .
$$

It is clear from this expression that the instanton size plays a role of the UV cutoff, since it provides an exponential suppression of the integrand at large $(\alpha, \beta)$ what corresponds to the large momentum. Computing this integral we have

$$
\begin{aligned}
D_{I}(0 ; \varepsilon, \lambda, \rho)= & -\frac{\rho^{4} \pi^{2}}{4}\left(\rho^{2} \lambda^{2} \pi\right)^{\varepsilon} \frac{\Gamma(1-\varepsilon)}{\varepsilon} \\
& \times\left(1+\varepsilon \int_{0}^{1} \int_{0}^{1} \int_{0}^{1} d x d y d z\right. \\
& \left.\times \ln \left[\frac{x z+y(1-z)}{z(1-z)}\right]+O\left(\varepsilon^{2}\right)\right) .
\end{aligned}
$$

Applying the renormalization procedure as described in the previous section, we find in the leading order the instanton contribution to the renormalization constant

$$
Z_{I}(\gamma, \varepsilon)=1-2 h(\gamma)\left(\frac{1}{\varepsilon} \int d n(\rho) \frac{\rho^{4} \pi^{2}}{4}+\Delta_{\mathrm{NP}}\right)
$$

where the finite term is

$$
\Delta_{\mathrm{NP}}=\gamma_{E}+\ln \pi+\frac{\pi^{2}}{6}-\frac{3}{2}
$$

and obtain the corresponding contribution to the Wilson loop

$$
w_{I}^{(1)}(\gamma, \lambda)=1+\pi^{2} h(\gamma) \int d n(\rho) \rho^{4} \ln (\rho \lambda) .
$$

In order to estimate the magnitude of the instanton induced effect we consider the distribution function which has been suggested in Ref. [18] (and discussed in Ref. [9] in the framework of constrained instanton model) in order to describe the lattice data [6], namely, the following one:

$$
d n(\rho)=\frac{d \rho}{\rho^{5}} C_{N_{c}}\left(\frac{2 \pi}{\alpha_{S}(\rho)}\right)^{2 N_{c}} \exp \left(-\frac{2 \pi}{\alpha_{S}(\rho)}\right) \exp \left(-2 \pi \sigma \rho^{2}\right)
$$

where the numerical constant $C_{N_{c}}$ is determined by the number of colors

$$
C_{N_{c}}=\frac{0.466 e^{-1.679 N_{c}}}{\left(N_{c}-1\right) !\left(N_{c}-2\right) !} \approx 0.0015
$$

and the string tension is accepted to be $\sigma \approx(0.44 \mathrm{GeV})^{2}$ $[8,18]$. Then, using the one loop expression for the running coupling constant

$$
\alpha_{S}(\rho)=-\frac{2 \pi}{\beta_{0} \ln \rho \Lambda_{Q C D}}, \quad \beta_{0}=\frac{11 N_{c}-2 n_{f}}{3},
$$

we find the instanton contribution (40) in the form [in the distribution (41), the slow varying logarithmic factor due to the power of the coupling $\alpha_{S}$ is assumed to be constant, and taken at the point of the mean instanton size $\bar{\rho}$ ]

$$
\begin{aligned}
w_{I}^{(1)}(\gamma, \lambda)= & 1+\pi^{2} h(\gamma) \frac{C_{N_{c}} \Gamma\left(\beta_{0} / 2\right)}{4}\left(\frac{2 \pi}{\alpha_{S}(\bar{\rho})}\right)^{2 N_{c}} \\
& \times\left(\frac{\Lambda_{\mathrm{QCD}}}{\sqrt{2 \pi \sigma}}\right)^{\beta_{0}} \ln \frac{\lambda^{2}}{2 \pi \sigma},
\end{aligned}
$$

where we omitted the constant term on the right hand side since it can be easily removed by the finite renormalization.

\footnotetext{
${ }^{2}$ We consider the singular gauge, since in the regular gauges the profile function decreases insufficiently rapidly at infinity, and therefore the contribution of the infinitely distant part of the contour must be taken into account, in contrast to the singular gauge where this contribution vanishes.
} 
The expression (44) shows explicitly that the instantons yield the powerlike corrections to the perturbative result, which is expected from a general consideration. Indeed, the powerlike behavior of the nonperturbative corrections to the high energy QCD processes may be obtained from the renormalon analysis (see, e.g., Ref. [19]).

It is instructive to express the result (44) in terms of the mean instanton size $\bar{\rho}$ and the instanton density $\bar{n}$ calculated directly from the distribution (41), which read, respectively,

$\bar{\rho}=\frac{\Gamma\left(\beta_{0} / 2-3 / 2\right)}{\Gamma\left(\beta_{0} / 2-2\right)} \frac{1}{\sqrt{2 \pi \sigma}}$,

$\bar{n}=\frac{C_{N_{c}} \Gamma\left(\beta_{0} / 2-2\right)}{2}\left(\frac{2 \pi}{\alpha_{S}(\bar{\rho})}\right)^{2 N_{c}}\left(\frac{\Lambda_{\mathrm{QCD}}}{\sqrt{2 \pi \sigma}}\right)^{\beta_{0}}(2 \pi \sigma)^{2}$.

Numerically, these quantities correspond to the usual mean size $\rho_{0}$ and density $n_{0}$ of the instanton liquid model, which parametrize the deltalike approximation to the distribution (41) (this approach, which was proposed about two decades ago [20], now gets a strong support in lattice data [6]):

$$
d n(\rho)=n_{0} \delta\left(\rho-\rho_{0}\right) d \rho,
$$

where the parameters were estimated in Ref. [5]:

$$
n_{0} \approx 1 \mathrm{fm}^{-4}, \quad \rho_{0} \approx 1 / 3 \mathrm{fm} .
$$

To compare the instanton induced and perturbative parts, which are, respectively,

$$
\begin{aligned}
& w_{I}(\gamma)=1-K h(\gamma) \pi^{2} \bar{n} \bar{\rho}^{4}\left[\ln \frac{\mu^{2}}{\lambda^{2}}-\ln \bar{\rho}^{2} \mu^{2}\right] \\
& W_{P}(\gamma)=1-\frac{\alpha_{S}(\mu)}{2 \pi} C_{F} h(\gamma) \ln \frac{\mu^{2}}{\lambda^{2}},
\end{aligned}
$$

where

$$
K=\frac{\Gamma\left(\beta_{0} / 2\right)\left[\Gamma\left(\beta_{0} / 2-2\right)\right]^{3}}{2\left[\Gamma\left(\beta_{0} / 2-3 / 2\right)\right]^{4}} \approx 0.74,
$$

we assume that the factorization scale $\mu$ (which divides the soft and hard regions of momenta in the factorized quark form factor) is of order of the inverse instanton size $\mu$ $\approx \bar{\rho}^{-1} \approx 0.6 \mathrm{GeV}$. Then we write the total leading order contribution to the Wilson loop expectation value in the form

$$
\begin{aligned}
W\left(\gamma, \rho_{0} \lambda\right)=1 & +\frac{\alpha_{S}\left(\bar{\rho}^{-1}\right)}{2 \pi} C_{F} h(\gamma) \ln \left(\bar{\rho}^{2} \lambda^{2}\right) \\
& \times\left(1+K \frac{S_{0} \pi^{2} \bar{n} \bar{\rho}^{4}}{C_{F}}\right),
\end{aligned}
$$

where $S_{0}=8 \pi^{2} / g^{2}\left(\bar{\rho}^{-1}\right) \approx 10$ is the "classical enhancement" factor with the renormalized coupling constant $g(\mu)$ at the energy scale $\mu \approx \bar{\rho}^{-1}$. The ratio of the instanton correction to the perturbative leading term is given by

$$
\frac{\text { Instanton }}{\text { 1-loop perturb. }}=K \frac{S_{0} \pi^{2} \bar{n} \bar{\rho}^{4}}{C_{F}} \approx 0.5,
$$

which is estimated using the conventional value for the packing fraction $[20]$

$$
\pi^{2} \bar{n} \bar{\rho}^{4} \approx 0.1
$$

One can see using the main formula (44) that the strong power suppression of the instanton part is partially compensated by the large factor $S_{0}^{2 N_{c}+1}$. This means that at the energy scale of order of $\bar{\rho}^{-1}$ the magnitude of the instanton induced effects is comparable to the leading perturbative part, and must be taken into account as well.

Let us estimate now the two-loop perturbative contribution which may be equally important at the chosen low scale, where the strong coupling constant is not small enough $\left[\alpha_{S}(0.6 \mathrm{GeV}) \approx 0.5\right]$. The two-loop result has been obtained in Ref. [14] and in the limits of small and large angles is given by

$$
W^{(2)}(\gamma) \approx \begin{cases}\left(\frac{\alpha_{S}}{\pi}\right)^{2} C_{F} N_{c} \frac{\gamma^{2}}{3}\left[\frac{11}{48} \ln ^{2} \frac{\mu^{2}}{\lambda^{2}}-\left(\frac{3}{8}-\frac{\pi^{2}}{12}+\frac{67}{72}\right) \ln \frac{\mu^{2}}{\lambda^{2}}\right] & \text { for small } \gamma, \\ \left(\frac{\alpha_{S}}{\pi}\right)^{2} C_{F} N_{c} \gamma\left[\frac{11}{48} \ln ^{2} \frac{\mu^{2}}{\lambda^{2}}-\left(\frac{67}{72}-\frac{\pi^{2}}{24}\right) \ln \frac{\mu^{2}}{\lambda^{2}}\right] & \text { for large } \gamma\end{cases}
$$

From these expressions we get that the ratio of the instanton contribution to the perturbative two-loop term is approximately equal to 1.4 and varies slightly (only about 10\%) with changing of $\gamma$ over whole allowed kinematical interval. Thus, the complete consideration of the quark form factor at the low momentum scale must include both the two-loop perturbative part and the leading order instanton one, which appear to be of the same order of magnitude.

\section{EXPONENTIATION OF PERTURBATIVE AND INSTANTON CORRECTIONS}

Expressions (51), (54) define the first terms of the Wilson loop expansion in gauge fields. On the basis of the exponentiation theorem [17] for the non-Abelian path-ordered exponentials it is well known that perturbative corrections to the Sudakov form factor are exponentiated to high orders in the QCD coupling constant. The theorem states that the contour 
average $W_{P}(C)$ can be expressed as

$$
W_{P}(C)=\exp \left[\sum_{n=1}^{\infty}\left(\frac{\alpha_{S}}{\pi}\right)^{n} \sum_{W \in W(n)} C_{n}(W) F_{n}(W)\right],
$$

where summation in the exponential is over all diagrams $W$ of the set $W(n)$ of the two-particle irreducible contour averages of $n$th order of the perturbative expansion. The coefficients $C_{n}(W) \propto C_{F} N_{c}^{n-1}$ are the "maximally non-Abelian" parts of the color factor corresponding to the contribution coming from a diagram $W$ to the total expression (55) in the contour gauge, and the factor $F_{n}(W)$ is the contour integral presented in the expression for $W$. This means that the essential diagrams are only those, which do not contain the lowerorder contributions as subgraphs and, as a result, the higherorder terms are non-Abelian.

Let us now demonstrate how the single instanton contribution is exponentiated in the small instanton density parameter, treating the instanton vacuum as a dilute medium [21]. The gauge field is taken to be the sum of individual instanton fields in the singular gauge (24), (35), with their centers at the points $z_{j}$ 's. In this gauge, the instanton fields fall off rapidly at infinity, so the instantons may be considered individually in their effect on the loop. Moreover, the contribution of infinitely distant parts of the contour may be neglected and only those instantons will influence the loop integral, which occupy regions of space-time intersecting with the quark trajectories. Since the parametrization of the loop integral along rays of the angle plays the role of the proper time, a time-ordered series of instantons arises and has an effect on the Wilson loop. Thus, the contribution of $n$ instantons to the loop integral $W_{I}(\gamma)$ can be written in the dilute approximation as

$$
W_{I}^{(n)}(\gamma)=\operatorname{Tr}\left(U^{1} U^{2} \cdots U^{n} U^{n \dagger} \cdots U^{2 \dagger} U^{1 \dagger}\right),
$$

where the ordered line integrals $U_{i}$ 's

$$
\begin{aligned}
U^{j}(\gamma)= & T\left\{\exp \left(i g \int_{0}^{\infty} d \sigma v_{1}^{\mu} A_{\mu}\left(v_{1} \sigma-z_{j}\right)\right)\right. \\
& \left.\times \exp \left(i g \int_{-\infty}^{0} d \tau v_{2}^{\mu} A_{\mu}\left(v_{2} \tau-z_{j}\right)\right)\right\}
\end{aligned}
$$

are associated with individual instantons with the positions $z_{j}$ 's. Because of the wide separation of the instantons in the dilute phase and rapid falloff of fields in the singular gauge, the upper and lower limits of the line integrals are extended to infinity. The line integrals $U^{i \dagger}$ 's take into account the part of the contour that goes at infinity from $+\infty$ back to $-\infty$ and in the singular gauge $U^{i \dagger}=1$. For $U^{j}\left(U^{j \dagger}\right)$, the integral is taken over the increasing (decreasing) time piece of the loop.

Then, the expression is simplified when averaging over the gauge orientations of instantons. The averaging is reduced to substitution of $U^{j}$ by $g_{j} U^{j} g_{j}^{-1}$, where $g_{j}$ is an element of color group, and independent integration of each $g_{j}$ over the properly normalized group measure is performed. Under this averaging one gets

$$
U^{n} U^{n \dagger} \rightarrow \frac{1}{N_{c}} \operatorname{Tr}\left(U^{n} U^{n \dagger}\right),
$$

which is just the single instanton contribution $w_{I}^{(n)}(\gamma)$ as it is given by Eqs. (25), (28). But then if the averaging is done in the inverse order, from $n$ down to 1 , the entire loop integral collapses to a product of traces

$$
W_{I}^{(n)}(\gamma) \rightarrow \lim _{n \rightarrow \infty} \prod_{j=1}^{n} w_{I}^{(j)}(\gamma) .
$$

Since the individual instantons are considered to be decoupled in the dilute medium, the total multiple instanton contribution to the vacuum average of the Wilson operator simply exponentiates the all-order single instanton term $w_{I}(\gamma)$ in Eq. (28), and one has

$$
W_{I}(\gamma)=\lim _{n \rightarrow \infty}\left\{1+\frac{1}{n} w_{I}(\gamma)\right\}^{n}=\exp \left[w_{I}(\gamma)\right] .
$$

Thus, we have proved that in the dilute regime, the full instanton contribution to the quark form factor is given by the exponent of the all-order single instanton result. The exponentiation arises due to taking into account the manyinstanton configurations effect. As it is well known, in QED there occurs the exponentiation of the one-loop result due to Abelian character of the theory. In the instanton case, the analogous result takes place since instantons belong to the $S U(2)$ subgroup of the $S U(3)$ color group and the pathordered exponents coincide with the ordinary ones. Exponentiation applied to the lowest order terms (51), (54) leads to vanishing the Wilson loop as a power of an IR cutoff $\lambda$. Then, the additional instanton contributions suggest that the Wilson loop decreases in the instanton medium faster with $\lambda$ than in purely perturbative theory.

\section{CONCLUSION}

We have calculated the instanton contribution to the soft part of the quark form factor, described in terms of the vacuum expectation value of the Wilson loop for the contour of a special form (28), (29). Further analysis of high energy behavior of this form factor must take into account the contributions of the hard and collinear parts as well. In order to do this, we would need to "free" the scale $\mu$ and consider it as a factorization parameter lying between the hard scale $Q$ and the IR cutoff $\lambda(\lambda<\mu<Q)$. Then we would find that the instantonic part, being UV finite due to the finite instanton size, does not contain an explicit dependence on the UV cutoff $\mu$, and therefore does not contribute to the cusp anomalous dimension. However, this issue is out of scope of the present paper, and we did not address this question here.

The situation may be changed essentially in some other hadronic hard processes. For example, the Wilson path for (in)elastic high energy quark-quark scattering consists of two infinite straight lines lying in two different planes, separated by the finite transverse distance $[3,8]$. The calculation of the Wilson operator for this contour reduces formally to the 
evaluation of the path integrals analogous to the ones considered above. The only difference is that the sides of these contours would belong to the different planes. The transverse distance between them provides then an UV cutoff for the integrals, both for perturbative and instanton fields, and the parameter of the dimensional regularization $\lambda$ becomes the only (IR) cutoff for them. Therefore, the corresponding anomalous dimension would contain the instanton induced part, and the instantons would play a nontrivial role in the solution of the corresponding renormalization group equations. This topic will be a subject of the forthcoming study.

Moreover, in the instanton calculations of the high energy parton scattering processes [8], the integrals over the instanton field can be computed in all orders by virtue of the infinite length of lines that form the integration path specific for this process. The latter fact leads to the trivial factorization of the $\gamma$-angle dependence from the corresponding Wilson integral, and extract the instanton contribution into the numerically calculable form factors. In our case, one deals with the cusp, rather than cross singularities. The all-order calculation given formally by Eqs. (28), (29) is quite complicated for the contour with cusp, so we postpone the solution of this task.

To summarize, within the instanton vacuum model we have developed an approach which allows one to calculate the nonperturbative contributions to the Wilson integrals over the infinite contour with a cusp that represent, e.g., the classical trajectories of partons participating in hard collisions. We have proved that in the dilute regime, the full instanton contribution to the quark form factor is given by the exponentiated all-order single instanton result, see Eq. (59). In the weak-field limit, the instanton contribution to the soft part of the color singlet quark form factor is found explicitly in terms of the instanton profile function in the singular gauge. It is shown that the instanton induced effects are of a power type (44), but nevertheless they are comparable in magnitude to the perturbative ones at the scale of order of the inverse average size of the instanton in the instanton vacuum, see Eq. (51).

\section{ACKNOWLEDGMENTS}

We are grateful to D. Antonov, N.I. Kochelev, S.V. Mikhailov, and S. Tafat for fruitful discussions. This work was partially supported by RFBR (Grant Nos. 02-02-16194, 01-02-16431) and INTAS (Grant No. 00-00-366). The work of I.Ch. was also supported by RFBR Grant No. 00-1596577. He is grateful to the Abdus Salam ICTP in Trieste for the kind invitation and hospitality, where a part of this work was done.

\section{APPENDIX}

Below we write down some useful formulas that have been used in the calculations. The dimensionally regularized free propagator in the Euclidean space reads $(n=4-2 \varepsilon)$ :

$$
\begin{aligned}
D_{\mu \nu}(z ; \xi)= & \lambda^{4-n} \int \frac{d^{n} k}{(2 \pi)^{n}} e^{-i k z}\left(\frac{\delta_{\mu \nu}}{k^{2}}-\xi \frac{k_{\mu} k_{\nu}}{k^{4}}\right) \\
= & \frac{\left(\lambda^{2} \pi\right)^{(2-n / 2)}}{4 \pi^{2}} \Gamma(n / 2-1)\left(\delta_{\mu \nu} \frac{(1-\xi / 2)}{z^{2(n / 2-1)}}\right. \\
& \left.-\xi z_{\mu} z_{\nu} \frac{(n / 2-1)}{z^{2(n / 2)}}\right)
\end{aligned}
$$

The form factors $d_{1,2}(u)$ defined in Eq. (10) are given by $\left(u=z^{2}\right)$

$$
d_{1}(u)=\frac{\left(\lambda^{2} \pi\right)^{(2-n / 2)}}{16 \pi^{2}} \frac{\Gamma(n / 2-2)}{u^{(n / 2-2)}}, \quad d_{2}(u)=\xi d_{1}(u) .
$$

The partial derivatives in terms of the derivative with respect to the interval $u$ in $n$-dimensional space-time

$$
\begin{gathered}
\partial_{z}^{2}=2 n \partial_{u}+4 u \partial_{u}^{2}, \\
\partial_{\mu} \partial_{\nu}=2 \delta_{\mu \nu} \partial_{u}+4 z_{\mu} z_{\nu} \partial_{u}^{2} .
\end{gathered}
$$

Scalar products in the Euclidean space

$$
v_{\mu}^{1} v_{\nu}^{2} \delta_{\mu \nu}=\cos \gamma, \quad v_{\mu}^{1} v_{\nu}^{2} z_{\mu} z_{\nu}=z^{2} \cos \gamma+s t \sin ^{2} \gamma .
$$

For calculations of the perturbative part we used the following integrals:

$$
\int_{0}^{\infty} d s d t e^{-\alpha\left(s^{2}+t^{2}+2 s t \cos \gamma\right)}=\frac{1}{2 \alpha} \frac{\gamma}{\sin \gamma}
$$

and

$$
\int_{0}^{\infty} d s d t s t e^{-\alpha\left(s^{2}+t^{2}+2 s t \cos \gamma\right)}=\frac{1-\gamma \cot \gamma}{4 \alpha^{2} \sin ^{2} \gamma} .
$$

Therefore, one has for a function $d(u)$ :

$$
d_{i}^{\{k\}}(u)=(-)^{k} \int_{0}^{\infty} d \alpha \alpha^{k} e^{-\alpha u} \bar{d}_{i}(\alpha)
$$

and gets

$$
\begin{gathered}
\int_{0}^{\infty} d s d t d^{\prime}(u)=-\frac{\gamma}{2 \sin \gamma} d(0), \\
\int_{0}^{\infty} d s d t u d^{\prime \prime}(u)=\frac{\gamma}{2 \sin \gamma} d(0), \\
\int_{0}^{\infty} d s d t \operatorname{std}^{\prime \prime}(u)=\frac{1-\gamma \cot \gamma}{4 \sin ^{2} \gamma} d(0) .
\end{gathered}
$$


[1] Yu.M. Makeenko and A.A. Migdal, Phys. Lett. 88B, 135 (1979); Nucl. Phys. B188, 269 (1981); A. Polyakov, Gauge Fields and Strings (Harwood Academic, Chur, Switzerland, 1987).

[2] A. Bassetto, M. Ciafaloni, and G. Marchesini, Phys. Rep. 100, 201 (1983); G. Korchemsky, Phys. Lett. B 220, 629 (1989); G. Korchemsky and G. Sterman, Nucl. Phys. B437, 415 (1995); S. Tafat, J. High Energy Phys. 05, 004 (2001); G. Korchemsky and A. Radyushkin, Phys. Lett. B 171, 459 (1986).

[3] O. Nachtmann, Ann. Phys. (N.Y.) 209, 436 (1991).

[4] G. Korchemsky and A. Radyushkin, Phys. Lett. B 279, 359 (1992).

[5] T. Schäfer and E.V. Shuryak, Rev. Mod. Phys. 70, 323 (1998).

[6] D.A. Smith and M.J. Teper, Phys. Rev. D 58, 014505 (1998); J.W. Negele, Nucl. Phys. B (Proc. Suppl.) 73, 92 (1999).

[7] S. Moch, A. Ringwald, and F. Schrempp, Nucl. Phys. B507, 134 (1997).

[8] E. Shuryak and I. Zahed, Phys. Rev. D 62, 085014 (2000); M. Nowak, E. Shuryak, and I. Zahed, ibid. 64, 034008 (2001).

[9] A.E. Dorokhov, S.V. Esaibegyan, and S.V. Mikhailov, Phys. Rev. D 56, 4062 (1997); A.E. Dorokhov, S.V. Esaibegyan, A.E. Maximov, and S.V. Mikhailov, Eur. Phys. J. C 13, 331 (2000).
[10] G. Korchemsky, Phys. Lett. B 217, 330 (1989).

[11] V. Dotsenko and S. Vergeles, Nucl. Phys. B169, 527 (1980); A. Polyakov, ibid. B164, 171 (1980).

[12] R.A. Brandt, F. Neri, and M.-A. Sato, Phys. Rev. D 24, 879 (1981); R.A. Brandt, A. Gocksch, M.A. Sato, and F. Neri, ibid. 26, 3611 (1982).

[13] G. Korchemsky, Phys. Lett. B 325, 459 (1994).

[14] G. Korchemsky and A. Radyushkin, Nucl. Phys. B283, 342 (1987).

[15] E. Meggiolaro, Phys. Rev. D 53, 3835 (1996).

[16] N.S. Craigie and H. Dorn, Nucl. Phys. B185, 204 (1981); D. Knauss and K. Scharnhorst, Ann. Phys. (Leipzig) 41, 331 (1984).

[17] J.G.M. Gatheral, Phys. Lett. 133B, 90 (1983); J. Frenkel and J.C. Taylor, Nucl. Phys. B246, 231 (1984).

[18] E. Shuryak, hep-ph/9909458.

[19] M. Beneke, Phys. Rep. 317, 1 (1999).

[20] E. Shuryak, Nucl. Phys. B203, 93 (1982); B203, 116 (1982); B203, 140 (1982)

[21] C.G. Callan, R. Dashen, and D.J. Gross, Phys. Rev. D 17, 2717 (1978). 